\title{
Correlated lateral phase separations in stacks of lipid membranes
}

\author{
Takuma Hoshino ${ }^{1,2,3}$ * Shigeyuki Komura ${ }^{1,3}$ † and David Andelman ${ }^{2,37}$ \\ ${ }^{1}$ Department of Chemistry, Graduate School of Science and Engineering, \\ Tokyo Metropolitan University, Tokyo 192-0397, Japan \\ ${ }^{2}$ Raymond and Beverly Sackler School of Physics and Astronomy, \\ Tel Aviv University, Ramat Aviv, Tel Aviv 69978, Israel \\ ${ }^{3}$ Kavli Institute for Theoretical Physics China, CAS, Beijing 100190, China
}

(Dated: September 18, 2021)

\begin{abstract}
Motivated by the experimental study of Tayebi et al. [Nature Mater. 11, 1074 (2012)] on phase separation of stacked multi-component lipid bilayers, we propose a model composed of stacked two-dimensional Ising spins. We study both its static and dynamical features using Monte Carlo simulations with Kawasaki spin exchange dynamics that conserves the order parameter. We show that at thermodynamical equilibrium, due to strong inter-layer correlations, the system forms a continuous columnar structure for any finite interaction across adjacent layers. Furthermore, the phase separation shows a faster dynamics as the inter-layer interaction is increased. This temporal behavior is mainly due to an effective deeper temperature quench because of the larger value of the critical temperature, $T_{\mathrm{c}}$, for larger inter-layer interaction. When the temperature ratio, $T / T_{\mathrm{c}}$, is kept fixed, the temporal growth exponent does not increase and even slightly decreases as function of the increased inter-layer interaction.
\end{abstract}

\section{INTRODUCTION}

Biological membranes are constructed out of two monolayers (leaflets) arranged in a back-to-back configuration. They are mainly composed of phospholipids but contain also other molecules such as cholesterol, glycosugars, and proteins 1. In living organisms, these membranes can form multi-lamellar stacks known as lamellar bodies [2]. Examples of such highly folded membranous structures are thylakoid membranes of photosynthetic cyanobacteria or plant chloroplasts, and stratum corneum of human skin. Since multilamellar structures can combine single membrane functions in series, they offer possibilities for novel applications in photonics and as bio-sensors.

Over the last decade, many studies have been performed on artificial giant unilamellar vesicles (GUVs) composed of ternary mixtures of saturated lipid such as sphingomyelin, unsaturated lipid such as DOPC (1,2dioleoyl-sn-glycero-3-phosphocholine) and cholesterol [3. 4. By decreasing temperature, these ternary mixtures undergo a lateral phase separation, where a liquiddisordered $\left(\mathrm{L}_{\mathrm{d}}\right)$ phase coexists with a liquid-ordered $\left(\mathrm{L}_{\mathrm{o}}\right)$ one. It is known that the $\mathrm{L}_{\mathrm{o}}$ phase is rich in saturated lipid and cholesterol, while the $\mathrm{L}_{\mathrm{d}}$ phase is rich in the unsaturated lipid.

In a recent experimental study, Tayebi et al. 50 reported that a stack (typically composed of several hundred layers) of multicomponent lipid bilayers with phaseseparated domains exhibits inter-layer columnar ordering. Using ternary mixtures of sphingomyelin, DOPC and cholesterol, it was observed that domains in stacked

\footnotetext{
* hoshino-takuma@ed.tmu.ac.jp

$\dagger$ komura@tmu.ac.jp

$\ddagger$ andelman@post.tau.ac.il
}

bilayers align one on top of the other, thereby forming an uninterrupted columnar ordering across hundreds of bilayer membranes. Such a cooperative multilayer epitaxy was attributed to the interplay between intra-layer domain growth and inter-layer coupling. The formation of columnar structures in stacked bilayers is important because it allows for electrical currents and transport processes to pass through many transmembrane channels in a cooperative and efficient manner. Other possible applications of the columnar ordering can be as templates for membrane protein crystallization, which is necessary for $\mathrm{X}$-ray structural analysis of membrane proteins incorporated in bilayers.

As far as the dynamics of phase separation in stacks of membranes is concerned, the temporal evolution of the average inplane domain size, $R$, was shown to obey a power-law growth, $R \sim t^{\alpha}$ with $\alpha \approx 0.455$ [5]. This exponent is larger than the value obtained using GUVs with a single bilayer, for which the reported experimental value is $\alpha \approx 0.28 \pm 0.05[6$. Hence, Tayebi et al. concluded that the inplane domain growth in each of the bilayers of the stack is faster, as compared to the domain growth in GUVs.

In a subsequent paper [7, a model based on regular solution theory, which takes into account the inter-lamellar coupling of inplane phase-separated domains, was proposed. The calculated phase diagram was presented in terms of intra-layer and inter-layer coupling parameters, and contains three different regions: (i) a "one-phase" region in which the system does not exhibit phase separation; (ii) a "two-phase" region in which two phases coexist and domains in different layers along the normal $z$ direction are completely aligned and have the same composition in the various layers, and (iii) a "multi-phase" region in which there are unaligned inplane domains with different composition in the different layers. According to Ref. [7], the transition line between the "two-phase" and 
"multi-phase" regions strongly depends on the number of layers in the stack which was varied up to ten layers.

(a)
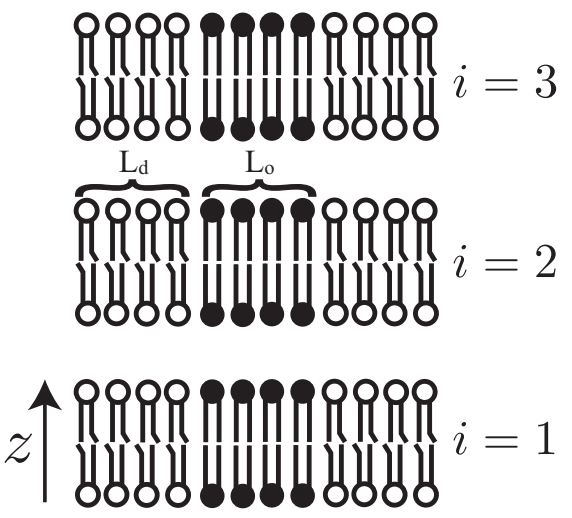

(b)

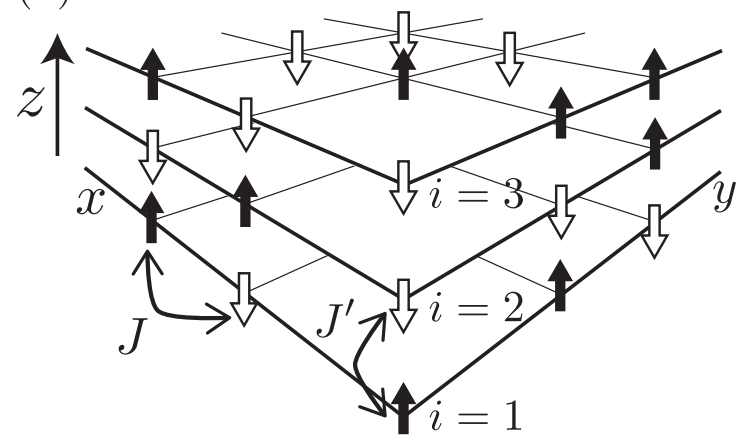

FIG. 1. (a) Schematic illustration of a stack of binary membranes, taken here as a stack of three bilayers in the $z$-direction. Each bilayer is composed of two identical leaflets containing saturated lipids ( $A$, black) and unsaturated lipids (B, white). Saturated and unsaturated lipids typically form $L_{o}$ and $L_{d}$ phases, respectively. As the lipid molecules are not allowed to exchange between different bilayers, their composition in each bilayer is fixed. (b) The stacked two-dimensional (2d) Ising model. Here the bilayer structure of each membrane is neglected. Lipid A and $B$ correspond to spin up (black) and spin down (white), respectively. $J$ is the coupling parameter between nearest-neighbor spins in the same layer, while $J^{\prime}$ is the coupling parameter between spins belonging to two nearest-neighboring layers.

Being motivated by these works [5, 7], we investigate the correlation between lateral phase separation in a stack of multi-layer membranes using a spin model called the stacked two-dimensional (2d) Ising model. This is the simplest model to describe a stack of binary membranes composed of two types of lipids. The model is the same as the anisotropic three-dimensional (3d) Ising model for a finite stack in the $z$-direction. The important difference between the two models is that in the former the order parameter (magnetization) in each layer is conserved. This requirement is based on the experimental fact that the A/B lipid composition in each layer almost does not change during experimental times.

In our model, we study the thermodynamical equilibrium features using Monte Carlo (MC) simulations. The main reason that we performed MC simulations rather than analyzing the mean-field free energy describing phase separation (as studied in Ref. 7), is to allow us to investigate the role of thermal fluctuations on the inter-layer domain correlation in stacked membranes. We show that the domains in each layer are correlated along the vertical $z$-direction, for any finite value of the interlayer interaction is positive, i.e., $J^{\prime}>0$. Hence, the system is either in a one- or two-phase state in equilibrium, and in our model the "multi-phase" state is not obtained in the thermodynamic limit of infinite lateral size, as long as the inter-layer coupling $J^{\prime}>0$. As anticipated, it is found that the phase-transition temperature, $T_{\mathrm{c}}\left(J^{\prime}\right)$, increases as function of the inter-layer interaction parameter.

We also investigate the dynamics of phase separation at fixed temperature $T$ in the two-phase coexistence region. We show that the accelerated temporal behavior of the phase separation for the stack is mainly driven by the increase of the temperature quench, $\Delta T=T_{\mathrm{c}}\left(J^{\prime}\right)-T$, because $T_{\mathrm{c}}\left(J^{\prime}\right)$ becomes larger for larger $J^{\prime}$. However, if the ratio $T / T_{\mathrm{c}}\left(J^{\prime}\right)$ is kept fixed, the dynamics of the phase separation is actually slower for larger values of the inter-layer coupling, $J^{\prime}$.

In the next section, we describe the stacked $2 \mathrm{~d}$ Ising model and review the MC simulation method. In Sec. III. we present the equilibrium properties of the model, and discuss the condition for domain columnar ordering. Section IV]describes the dynamics of domain growth for different values of the inter-layer interaction, and it is compared with a previous theoretical work.

\section{MODEL AND SIMULATION TECHNIQUE}

In our simulations, we use the stacked $2 d$ Ising model, shown in Fig. 1(a). We consider a stack of twocomponent lipid bilayer membranes composed of an A/B lipid mixture, although the experimental systems often consist of ternary lipid/cholesterol mixtures. This simplification does not affect the essential feature of the lateral phase separation. Another simplification is that we treat only symmetric bilayers where the composition of the two leaflets is identical. Hence, each lipid bilayer having a finite thickness can be mapped into a $2 \mathrm{~d}$ Ising model with conserved magnetization, expressing the fact that no lipid is allowed to exchange across layers. The $2 \mathrm{~d}$ Ising layers are stacked in the $z$-direction, and they interact with their two nearest-neighboring layers, as depicted in Fig. 1 (b).

The Hamiltonian of this stacked and coupled 2d Ising system can be written as:

$$
\begin{aligned}
H= & -J \sum_{i,\left\langle\boldsymbol{\rho}, \boldsymbol{\rho}^{\prime}\right\rangle} S_{i, \boldsymbol{\rho}} S_{i, \boldsymbol{\rho}^{\prime}}-J^{\prime} \sum_{i, \boldsymbol{\rho}} S_{i, \boldsymbol{\rho}} S_{i+1, \boldsymbol{\rho}} \\
& -\sum_{i, \boldsymbol{\rho}} \mu_{i} S_{i, \boldsymbol{\rho}}
\end{aligned}
$$

where up/down values of the spin, $S_{i, \rho}= \pm 1$, at $\boldsymbol{\rho}=$ 

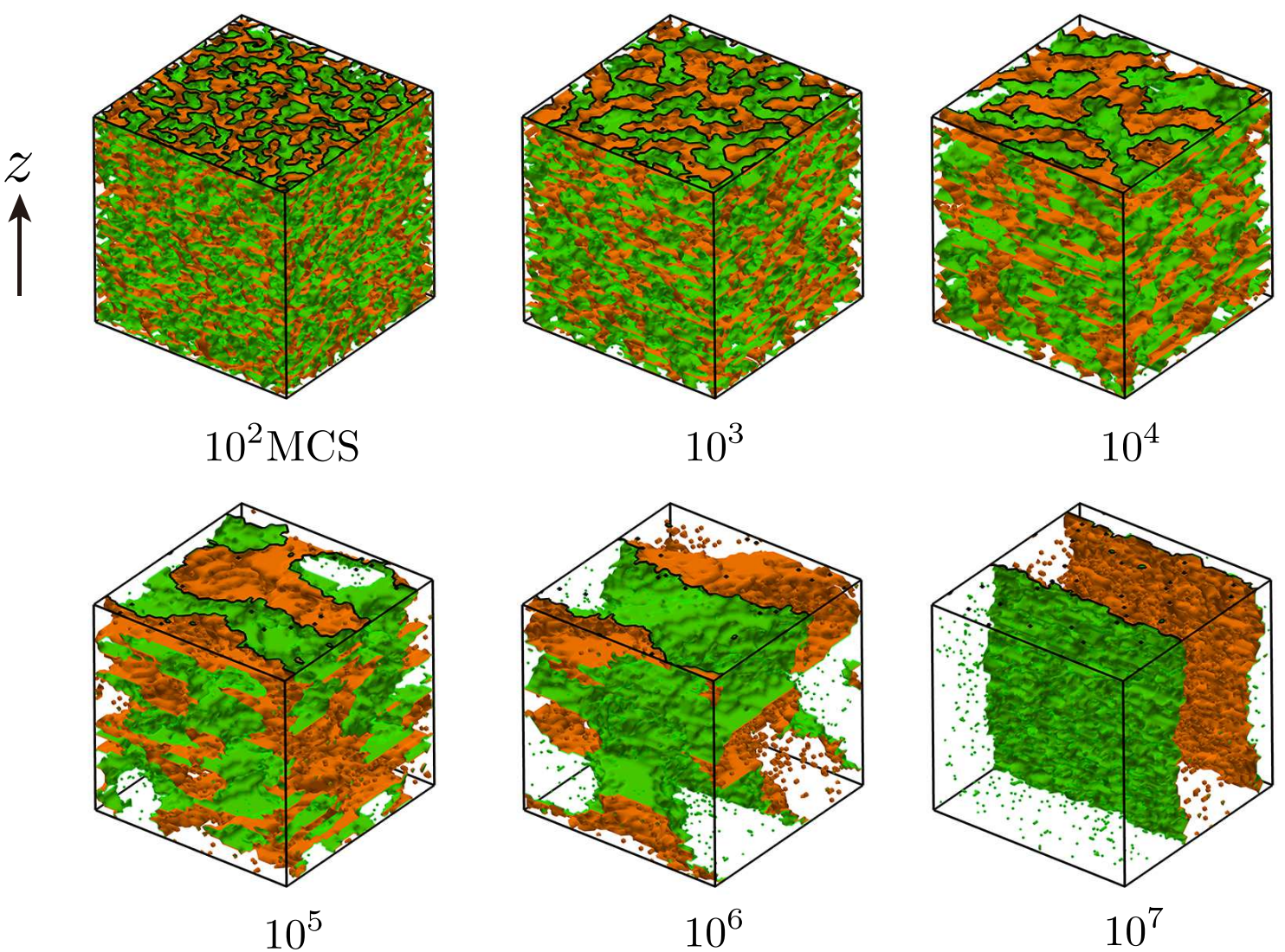

FIG. 2. Time evolution of phase separated domains in the stacked $2 \mathrm{~d}$ Ising model at different MC steps for $\lambda=0.1$ and $T / J=1.63$. The other parameters are $\bar{S}_{i, \rho}=0$ and $L=L_{z}=64$. For presentation purposes, only the interfaces of domain boundaries are shown, and the two different sides of the interfaces are represented by green and brown. The system is fully equilibrated after about $10^{7} \mathrm{MCS}$.

$(x, y)$ in the $i$-th layer corresponds to a lattice site occupied by an A or B lipids, respectively. The coupling between nearest-neighbor spins in the $x y$-plane (denoted by $\left.\left\langle\boldsymbol{\rho}, \boldsymbol{\rho}^{\prime}\right\rangle\right)$ is $J$, while the coupling with the nearestneighbor spins across layers in the $z$-direction is $J^{\prime}$. The physical origin of the inter-layer interaction $J^{\prime}$ is primarily attributed to direct van der Waals attractive interactions acting between neighboring bilayers 8. Other non-specific interactions, such as electrostatic and/or hydration interactions, can be taken into account through the second virial coefficient and will affect the value of $J^{\prime}$ as well 9, 10. Throughout this paper, we shall use the dimensionless ratio defined by $\lambda \equiv J^{\prime} / J$ as a measure of the inter-layer coupling strength.

In the above Hamiltonian, $\mu_{i}$ is the external field (chemical potential), which fixes the average magnetization (A/B composition) in the $i$-th layer. Although $\mu_{i}$ can, in general, take different values for different layers, we consider here the case where all of them are the same, $\mu_{i}=\mu$, fixing the same value of lipid composition in all layers. This assumption holds also for the dynamical states since we do not allow the lipids to be exchanged across different layers. The average order pa- rameter (A/B composition) in the $i$-th layer is denoted by $\bar{S}_{i, \rho}$, and throughout this paper (except in Fig. 5 (b)) we choose $\bar{S}_{i, \rho}=0$, which corresponds to a symmetric 1:1 A/B lipid mixture, i.e., at the critical composition. This is equivalent to setting the value of the chemical potential to zero, i.e., $\mu=0$.

The present model is related to the anisotropic $3 d$ Ising model for a finite slab. The special case of $\lambda=1$ corresponds to the isotropic 3d Ising model, whereas for $\lambda=0$ the stack is composed of non-interacting $2 \mathrm{~d}$ Ising layers. One interesting issue related to the anisotropic model, $0<\lambda<1$, is the crossover from $2 \mathrm{~d}$ to $3 \mathrm{~d}$ critical behavior 11 that will be explored below. We also note that the stacked $2 \mathrm{~d}$ Ising model has been studied a great deal in connection with multilayer adsorption phenomena on attractive substrates [12, 13, but not in the context of layers of binary mixtures with conserved magnetization (order parameter) as studied in this paper.

We investigate both the statics and dynamics of a stack of membranes based on the Hamiltonian presented in Eq. (1). We employ MC simulations for classical Ising spins on a finite $L \times L \times L_{z}$ lattice. Periodic boundary conditions are used in all three directions. The spin 
configurations are updated using Kawasaki exchange dynamics 14 in order to conserve the magnetization in each layer. This is based on the experimental fact that lipids almost do not exchange across different layers. Hence, their A/B inplane composition is fixed during experimental times.

The MC simulations presented here are performed in the following way. At each MC trial step, a site on the $3 \mathrm{~d}$ lattice and one of its nearest neighbors in the same layer are chosen at random. If the two spins are alike, a new site is again chosen at random. This process is repeated until two unlike nearest neighbor spins are found. Then, the probability of exchanging the two spins is determined by the standard Metropolis algorithm [15]. If the energy difference due to the spin exchange becomes negative, i.e., $\Delta E<0$, we accept the exchange. Otherwise, we accept the exchange with a probability $\exp (-\Delta E / T)$, where $T$ is the temperature and the Boltzmann constant, $k_{\mathrm{B}}$, was set to unity.

In one Monte Carlo step (MCS), this procedure is repeated $L \times L \times L_{z}$ times. The MC simulations are carried out by annealing the temperature gradually from an initial infinite temperature for which the spin configurations are completely disordered and uncorrelated. The first $10^{5}$ (or in some cases up to $10^{6}$ ) MCS are discarded in order to reach thermal equilibration. Furthermore, to avoid correlations between different equilibrated configurations, measurements are taken every $20 \mathrm{MCS}$, and we averaged over $10^{5}$ independent system configurations, in order to obtain sufficient statistics.

In order to investigate the phase separation dynamics, we monitor the domain coarsening as a function of time (MCS) at a constant temperature below $T_{\mathrm{c}}$. An example of a typical time evolution of phase separation is presented in Fig. 2 for $\lambda=0.1, T / J=1.63$ and $L=L_{z}=64$, where six snap-shots are shown from $10^{2}$ MCS till $10^{7} \mathrm{MCS}$. For clarity purposes, only the boundaries between domains of spin up (rich in lipid A) and spin down (rich in lipid B) are shown. In the initial time steps, the phase separation occurs inplane, and the domains coarsen without much out-of-plane coupling (due to the rather small value of $\lambda=0.1$ ). As time evolves, the inplane coarsening is also followed by out-ofplane columnar ordering, where the lipid A (and lipid B) rich domains are highly correlated along the $z$-direction. This is clearly seen for the fully equilibrated configuration occurring after about $10^{7} \mathrm{MCS}$ (last snap-shot). Here the two color boundaries, represent the two sides of the domain boundaries (while the inside of the domain is not shown). The boundaries look like extended interfaces separating inplane domains that are vertically connected along the $z$-direction, in agreement with experiment [5].
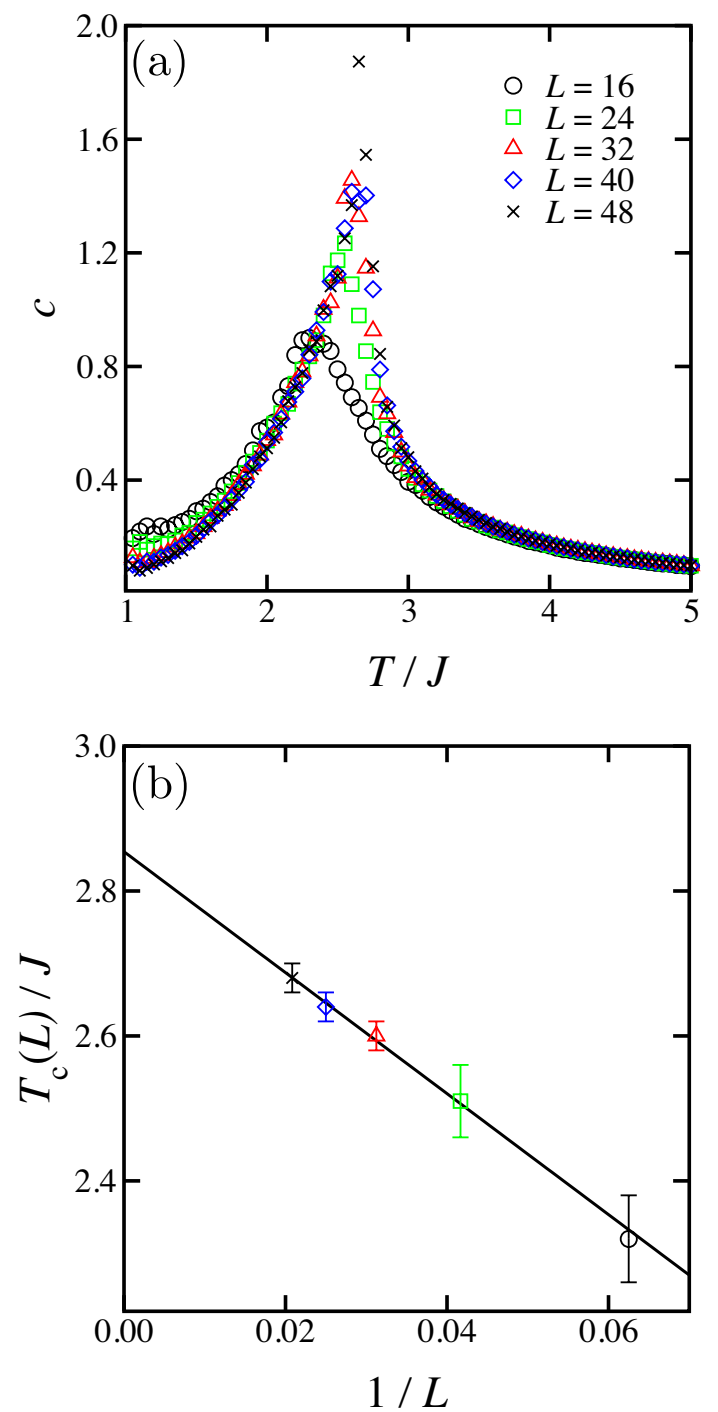

FIG. 3. (a) Specific heat per lattice site, $c$, as function of the dimensionless temperature $T / J$, for different lateral systemsize $L=16,24,32,40,48$. The other parameters are $\bar{S}_{i, \rho}=0$, $\lambda=0.1$ and $L_{z}=8$. For each system size, the peak position of $c$ is identified with an effective "phase transition" temperature. (b) Finite-size scaling analysis of the phase-transition temperature, $T_{\mathrm{c}}(L) / J$ for $\lambda=0.1$. The apparent phase-transition temperature is plotted as a function of $1 / L$. The solid line is the fit given by Eq. (3) with $\nu=1$ (see text). The extrapolated value for the critical temperature is $T_{\mathrm{c}}(\lambda=0.1) / J=2.85$.

\section{STATIC PROPERTIES OF THE STACKED DOMAINS}

In order to determine the phase-transition temperature and obtain the corresponding phase diagram, we compute the specific heat per lattice site defined as

$$
c=\frac{1}{L^{2} L_{z}} \frac{1}{T^{2}}\left(\left\langle H^{2}\right\rangle-\langle H\rangle^{2}\right),
$$


where $H$ is given by Eq. (1) and $\langle\cdots\rangle$ indicates an ensemble average. We note again that the above specific heat is calculated at constant magnetization (corresponding to constant lipid concentration in our model) of each layer. In our simulations, the ensemble average is taken by averaging over independent equilibrium spin configurations as explained in Sec. III. For a given system size and dimensionless ratio $\lambda$, we calculate $c$ as function of the dimensionless temperature $T / J$. Such a dependence of $c$ on $T / J$ is presented in Fig. 3(a) for several lateral system-sizes, $L$, and for $\lambda=0.1, L_{z}=8$, recalling that $L_{z}$ is the number of layers of the $3 \mathrm{~d}$ stack.

For each system size, we associate the peak position of the specific heat with the apparent critical temperature, $T_{\mathrm{c}}(L, \lambda)$, for a system with a finite size, $L$. Finite-size scaling analysis is then performed in order to determine the critical temperature for a slab of a finite $L_{z}$ layers in the thermodynamic limit $(L \rightarrow \infty)$. In Fig. 3(b), we plot $T_{\mathrm{c}}(L, \lambda=0.1)$ as a function of $1 / L$ for the same parameters as in (a). The plotted data are fitted with the following finite-size scaling assumption:

$$
T_{\mathrm{c}}(L, \lambda)=T_{\mathrm{c}}(\lambda)+a L^{-1 / \nu},
$$

where $T_{\mathrm{c}}(\lambda)=T_{\mathrm{c}}(L \rightarrow \infty, \lambda)$ is the infinite system critical temperature for a given $\lambda, a$ is a non-universal prefactor, and $\nu$ is the $2 \mathrm{~d}$ critical exponent for the correlation length in the $x y$-plane. We set $\nu=1$ in our analysis, following the work by Pham Phu et al. [16], who performed extensive MC simulations on magnetic Ising films (with $\lambda=1$ ) [17. We choose this $2 \mathrm{~d}$ critical exponent for the fitting because it was shown [16] that the $2 \mathrm{~d}$ character of the film is dominant even for $L_{z}=13$. The extrapolated critical temperature for $L \rightarrow \infty$ obtained from Fig. 3(b) is $T_{\mathrm{c}}(\lambda=0.1) / J=2.85$. We repeat this procedure for different values of the inter-layer interaction parameter in the range of $0 \leq \lambda \leq 1$, and determine the corresponding critical temperature, $T_{\mathrm{c}}(\lambda)$. We note that the value $\nu=1$ provides a good fitting for all the $\lambda$ values examined.

Somewhat surprisingly, finite-size effects in the $z$ direction are much weaker as compared to those in the lateral direction. This is shown in Fig. 4, where we plot $c$ as a function of $T / J$ when (a) $\lambda=0.1$, (b) $\lambda=0.5$ and (c) $\lambda=1$ for different number of layers, $L_{z}=4,8,12,16$, while the lateral size $L=48$ is kept fixed. For all $\lambda$ values studied here $(0.1 \leq \lambda \leq 1)$, the observed peak position: $T / J \approx 2.65$ in (a), 3.30 in (b) and 4.10 in (c), is almost independent of $L_{z}$, at least for $L_{z} \geq 8$. This means that, in our model with a fixed imposed magnetization (A/B composition) in each layer, the correlation in the $z$-direction is very strong due to the cooperative behavior of domains in different layers.

For fully equilibrated configurations, as shown in Fig. 2 after $10^{7}$ MCS, the domains are highly connected vertically along the $z$-direction, from the bottom layer to the top one. This is also shown in Fig. 5in which the columnar structure of domains in different layers is clearly shown. Hence, the correlation length in this direction exceeds $L_{z}$, and the constraint of fixed magnetization (A/B composition) in each layer induces a strong structural correlation in the $z$-direction even though the inter-layer interaction $J^{\prime}$ is smaller than the intra-layer interaction $J(\lambda \leq 1)$. A more quantitative argument for the domain connectivity will be given later. Because the number of layers, $L_{z}$, barely affects the MC results as shown in Fig. 4 for $\lambda=0.1,0.5$ and 1 , most of the simulations were done using $L_{z}=8$, which is sufficiently large in our case to observe the asymptotic behavior of $L_{z} \rightarrow \infty$. For the anisotropic $3 \mathrm{~d}$ Ising model without any constraint of conserved magnetization, as previously studied in Ref. [11, a very weak system-size dependence of the apparent critical temperature was observed by measuring the planar susceptibility.

The results of finite-size scaling analysis are shown in Fig. 6, where we plot $T_{\mathrm{c}}$ as a function of $\lambda$. The critical temperature interpolates between the $2 \mathrm{~d}$ and $3 \mathrm{~d}$ Ising results, $T_{\mathrm{c}}^{2 \mathrm{~d}}<T_{\mathrm{c}}(\lambda)<T_{\mathrm{c}}^{3 \mathrm{~d}}$; the exact value in $2 \mathrm{~d}$ (corresponding to $\lambda=0$ ) is known to be $T_{\mathrm{c}}^{2 \mathrm{~d}} / J=$ $2 / \ln (1+\sqrt{2}) \approx 2.269$ for square lattices [18, and the numerical estimate in $3 \mathrm{~d}$ (corresponding to $\lambda=1$ ) is $T_{\mathrm{c}}^{3 \mathrm{~d}} / J \approx 4.511$ for cubic lattices [19]. These two limits are recovered in our simulations and are seen in Fig. 6 for $\lambda=0$ and 1 , respectively. Although a more detailed $\lambda$-dependent scaling behavior of $T_{\mathrm{c}}(\lambda)$ was previously discussed in the limit of very small $\lambda[11,20$, we shall generalize the argument for the anisotropic case of finite $\lambda$, $0 \leq \lambda \leq 1$. When $T<T_{\mathrm{c}}(\lambda)$, the stack undergoes a phase separation, and the inplane domains rich in lipid A (spin up) are interconnected along the $z$-direction, bridging between adjacent layers and forming large connected domains of the same average composition. The same feature also occurs for the B-rich domains. Such a behavior can be clearly observed in Fig. 5.

In order to monitor quantitatively the degree of interconnectivity of domains in different layers, we define the following quantity:

$$
\delta^{2}=\frac{1}{L^{2}}\left\langle\sum_{\boldsymbol{\rho}}\left(\frac{1}{L_{z}} \sum_{i} S_{i, \boldsymbol{\rho}}-\bar{S}_{i, \boldsymbol{\rho}}\right)^{2}\right\rangle,
$$

where the average is taken over equilibrated MC configurations as explained above. This quantity can be cast also as:

$$
\delta^{2}=\frac{1}{L^{2} L_{z}^{2}} \sum_{\boldsymbol{\rho}} \sum_{i, j}\left\langle\left(S_{i, \boldsymbol{\rho}}-\bar{S}_{i, \boldsymbol{\rho}}\right)\left(S_{j, \boldsymbol{\rho}}-\bar{S}_{j, \boldsymbol{\rho}}\right)\right\rangle,
$$

and represents a special "magnetic susceptibility", where the correlations are taken only along the $z$-direction and then averaged laterally in each of the planes. When the domains are connected along the $z$-direction, the summation over different $i$-layers will produce a large value of $\delta$, while $\delta$ is small if the domains are uncorrelated across the layers even for $T<T_{\mathrm{c}}(\lambda)$. In Fig. 7), we plot $\delta^{2}$ as a function of $T / J$ for different values of $\lambda$, while fixing $L=16$ and $L_{z}=8$. Notice that even for $\lambda$ as small as 0.05 (blue diamonds), $\delta^{2}$ tends to increase as the temperature decreases below $T_{\mathrm{c}}(\lambda)$. This means that the 

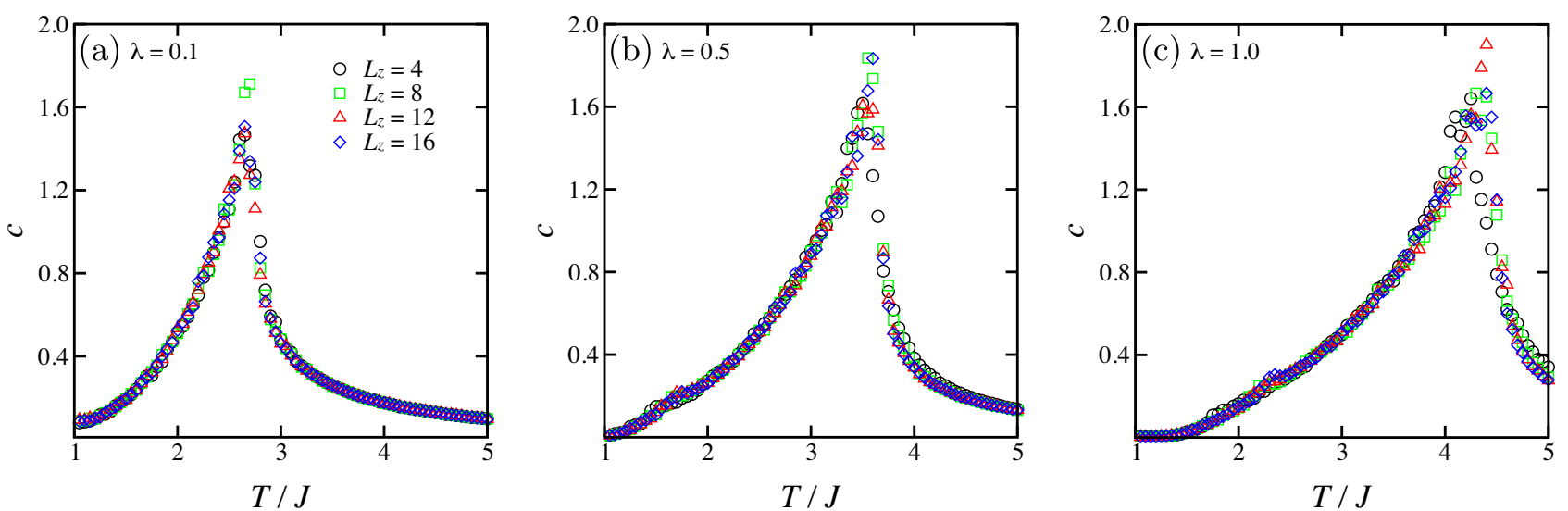

FIG. 4. Specific heat per lattice site, $c$, as function of the dimensionless temperature $T / J$, for different systems size $L_{z}=4,8,12,16$ for (a) $\lambda=0.1$, (b) $\lambda=0.5$ and (c) $\lambda=1$. The other parameters are $\bar{S}_{i, \rho}=0$ and $L=48$. The observed peak position: $T / J \approx 2.65$ in (a), 3.30 in (b) and 4.10 in (c), is almost independent of $L_{z}$, at least for $L_{z} \geq 8$.

(a)

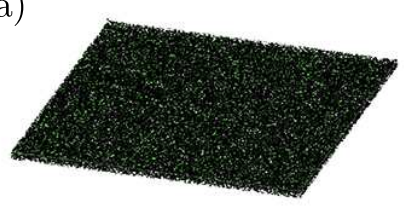

(b)
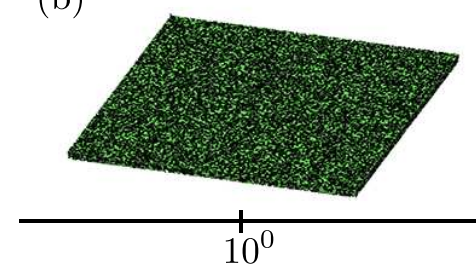
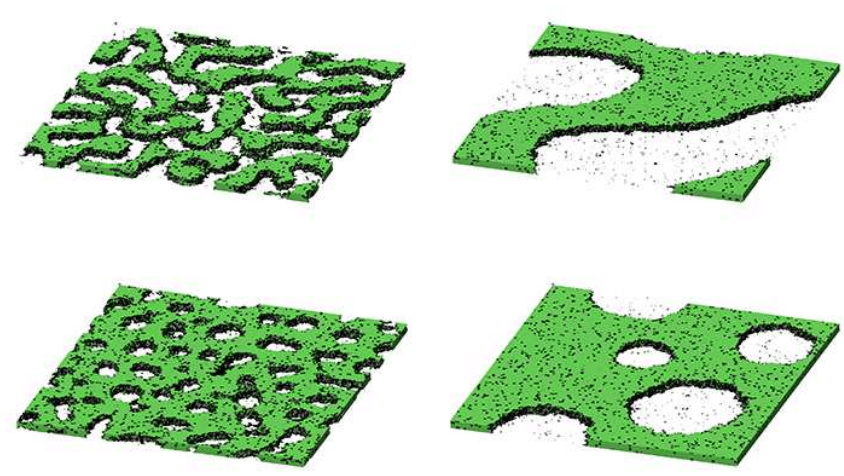

$10^{5}$

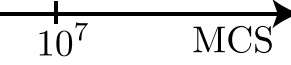

FIG. 5. Time evolution of phase-separated domains in a stacked 2d Ising model of eight layers, $L_{z}=8$, at different MC steps for (a) $\bar{S}_{i, \rho}=0$ and (b) $\bar{S}_{i, \rho}=0.4$. The other parameters are $\lambda=0.1, T / J=2.0$ and $L=256$.

domains are connected in the $z$-direction once the phase separation takes place. On the other hand, domains are independent and uncorrelated only when the inter-layer interaction is extremely small, i.e, $\lambda \leq 0.001$ in Fig. 7 . The situation is found to be marginal when $\lambda=0.01$ (red triangles) because $\delta^{2}$ then slightly deviates from zero at low temperatures.

Based on our MC results, we conclude that in the thermodynamic limit, $L \rightarrow \infty$, domains will always be connected for any finite inter-layer interaction, $J^{\prime}>0$. We give now a simple argument supporting this conclusion, and show that in the limit $L \rightarrow \infty$ but with a finite number of layers, $L_{z}$, the domains in different layers are uncorrelated only when $J^{\prime}=0(\lambda=0)$ is strictly obeyed. For the symmetric A/B case $\left(\bar{S}_{i, \rho}=0\right)$, each layer will eventually phase separate into two semi-infinite domains: one composed by the A lipid (spin up) and the other by the B lipid (spin down), as shown schematically in Fig. 8 . When the domains are fully correlated in the $z$-direction, as in Fig. 8(a), the total free energy of the stack consists of the contributions:

$$
F_{\text {con }}=-J^{\prime} L_{z} L^{2}+F_{\text {intra }},
$$

where $F_{\text {intra }}$ accounts for the intra-layer interactions. On the other hand, when the inplane domains are completely random and disconnected, as sketched in Fig. 8(b), the total free energy is dominated by an entropy contribution of arranging a random stack of $\mathrm{A}$ and $\mathrm{B}$ domains along the $z$-direction,

$$
F_{\text {dis }}=-T L_{z} \ln 2+F_{\text {intra }}
$$

with the same $F_{\text {intra }}$ as before because this term is common for both free energies. By comparing Eqs. (6) and (7), the threshold inter-layer interaction, $\left(J^{\prime}\right)^{*}$, separating the two states, is given by:

$$
\left(J^{\prime}\right)^{*}=\frac{T \ln 2}{L^{2}} .
$$




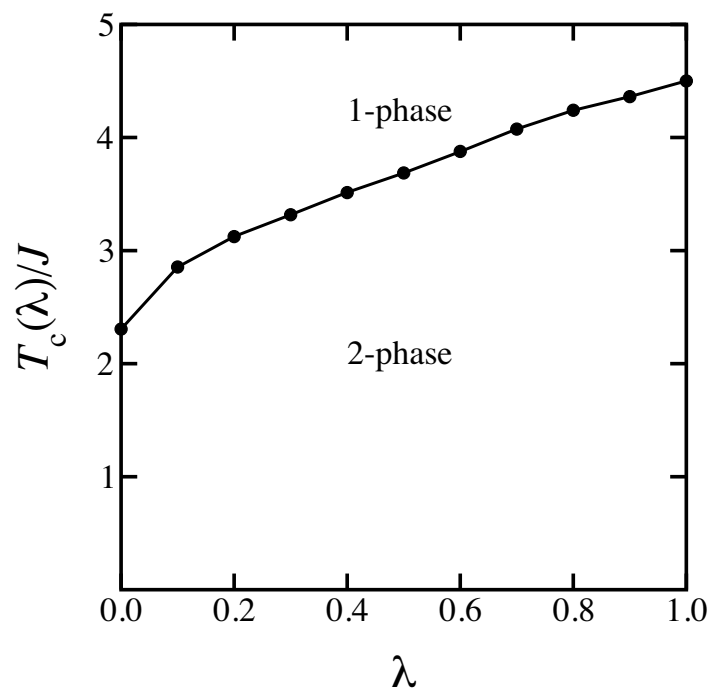

FIG. 6. The phase-separation temperature, $T_{\mathrm{c}}(\lambda) / J$, at the critical composition, as a function of the interaction parameter $\lambda$ for symmetric A/B mixtures, $\bar{S}_{i, \rho}=0$. The system is in a phase-separated state below the solid line, and in a one-phase state above the line.

Notice that $\left(J^{\prime}\right)^{*}$ depends on $L$ but not on $L_{z}$. For finite temperatures, it vanishes in the thermodynamic limit of $L \rightarrow \infty$. Hence, this simple scaling argument suggests that domains are always connected in the $z$-direction for any finite value of $J^{\prime}$. Therefore, for all $\lambda>0$, in the phase-separated region (below the critical temperature) presented in Fig. 6, domains should always form interconnected structures along the $z$-direction. As shown in Eqs. (6) and (7), the internal energy scales with $L^{2}$, while the entropy due to the random stacking of domains does not depend on $L$. Hence, the entropic effect can never overcome the internal energy in the thermodynamic limit, and leads to the stability of the columnar structure. This conclusion is not in agreement to that of Tayebi et al. [7, who claimed that there is a "multi-phase" state in which domains are not aligned and have different compositions even in thermodynamical equilibrium.

In the simulations, $\left(J^{\prime}\right)^{*}$ can be finite due to finite-size effects. For instance, if the temperature is chosen to be $T / J=1$ in Fig. 7, the threshold value for $L=16$ can be estimated as $\lambda^{*}=\left(J^{\prime}\right)^{*} / J \approx 2.7 \times 10^{-3}$. Since $\lambda=$ $10^{-2}$ (red triangles in Fig. 7) exceeds this threshold, the corresponding $\delta^{2}$ takes larger values at low temperatures. Moreover, the very weak finite-size effects along the $z$ direction is consistent with the lack of $L_{z}$-dependence of $\left(J^{\prime}\right)^{*}$ in Eq. (8).

\section{DYNAMICS OF PHASE SEPARATION}

We address now the effects of inter-layer interaction on the dynamics of phase separation as the system con-

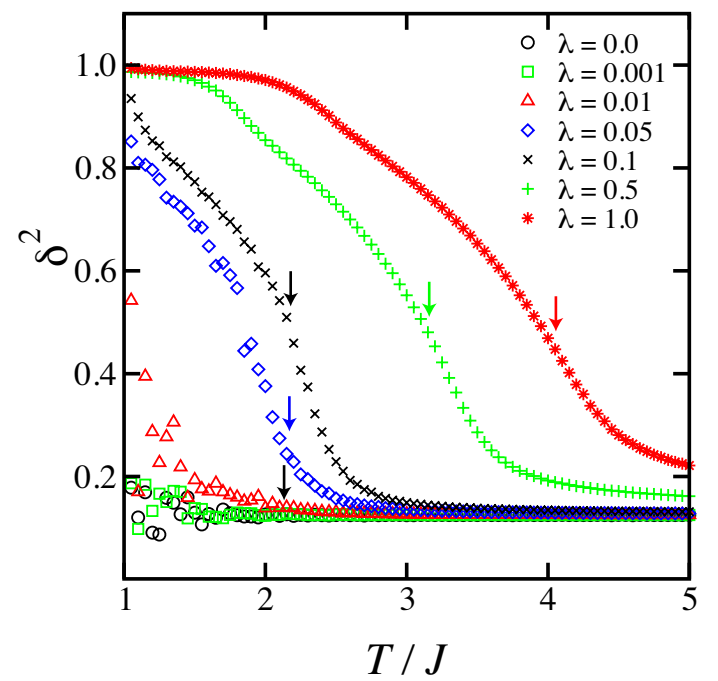

FIG. 7. The out-of-plane domain connectivity, $\delta^{2}$, defined in Eq. (4), as a function of the dimensionless temperature $T / J$, for different values of $\lambda=0,0.001,0.01,0.05,0.1,0.5,1.0$. The other parameters are $\bar{S}_{i, \rho}=0, L=16$ and $L_{z}=8$. The transition temperatures for different $\lambda$ values are indicated by arrows. The value of $\delta^{2}$ becomes larger when domains are correlated along the $z$-direction between different layers. This increase in $\delta^{2}$ is observed for lower temperatures and larger $\lambda$. (a)

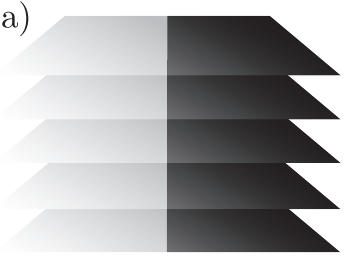

(b)

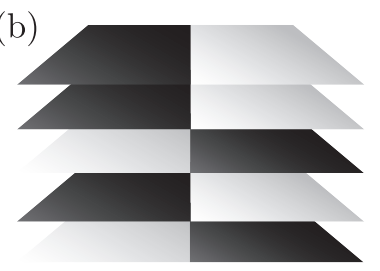

FIG. 8. Schematic representation of phase separated domains in a stack of membranes. Black and white domains are rich in A and B lipids, respectively. Two extreme cases are shown; (a) domains are fully connected in the $z$-direction, (b) domains are arranged at random and are disconnected.

verges towards its thermal equilibrium state. Under the assumption that scaling laws can be applied, the average domain size $R$ increases according to a temporal powerlaw: $R(t) \sim t^{\alpha}$ 4. For $2 \mathrm{~d}$ systems for which the total domain area is conserved, the average domain size $R$ is inversely proportional to the total interface length $\ell$, i.e., $R \sim \ell^{-1}$ [21, 22]. This can easily be seen because $R$ and $\ell$ are related by $\ell=2 \pi n R \sim n R$, where $n$ is the number of domains, and the total area of all domains, $A=\pi n R^{2} \sim n R^{2}$, is a conserved quantity. Hence, within the scaling hypothesis, the total interface length (in $2 \mathrm{~d}$ ) should behave as

$$
\ell(t) \sim t^{-\alpha}
$$

In our stacked Ising model, we calculate the interface length in each of the layers and average it over different 

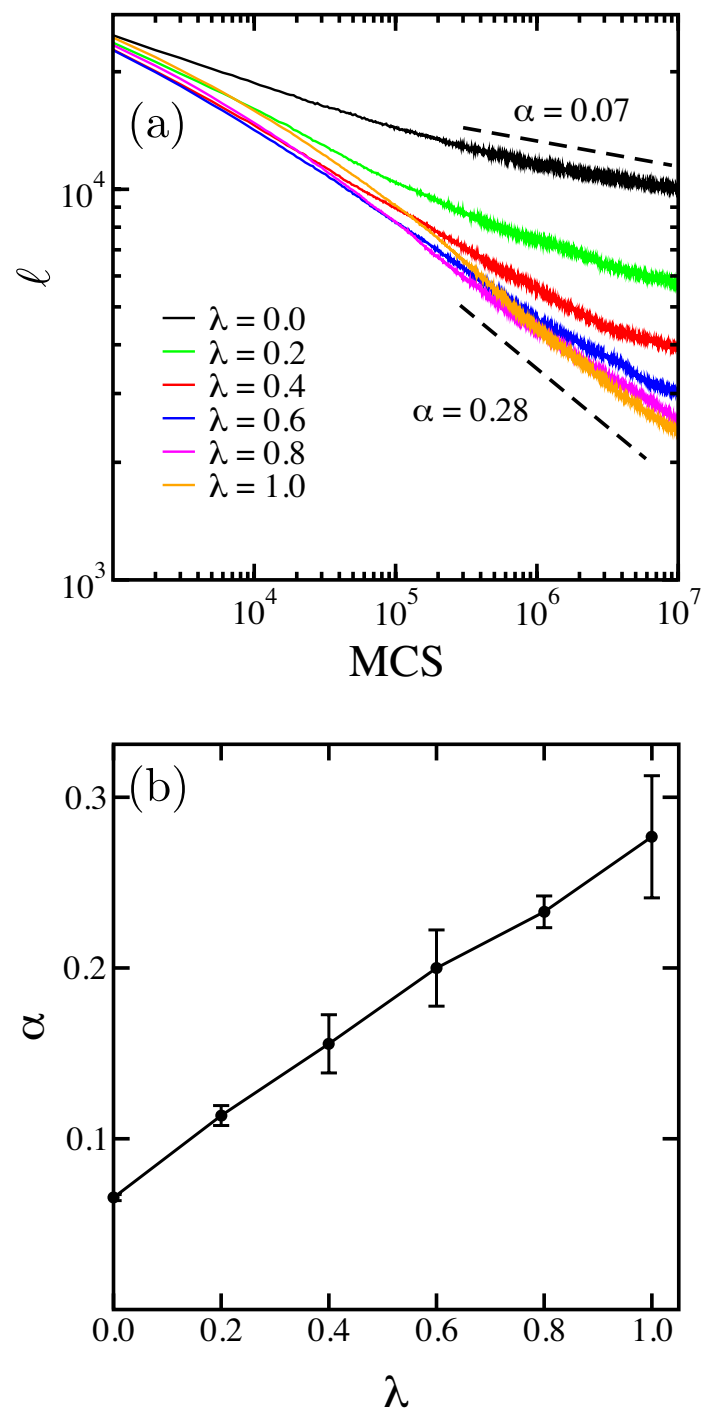

FIG. 9. (a) The temporal evolution of the total interface length $\ell$ as a function of time (MCS) for different values of $\lambda=0,0.2$, $0.4,0.6,0.8,1.0$, and for a temperature quench from the onephase state $(T \rightarrow \infty)$ into the two-phase state at $T / J=2.0$. The A/B mixture is symmetric, $\bar{S}_{i, \rho}=0, L=256$ and $L_{z}=8$. The average over three independent $M C$ runs is taken for each $\lambda$ value. The two dashed lines represent a power-law behavior with exponent $\alpha=0.07$ and 0.28 , which roughly bound the two limiting behaviors of the $\lambda$-dependent exponent, $\alpha$. (b) The domain growth exponent $\alpha$ as a function of $\lambda$, as obtained from (a).

layers. Note that the total interface length is proportional to the first term of the Hamiltonian in Eq. (1), which enumerates the number of bonds across the inplane $\mathrm{A} / \mathrm{B}$ interface.

In Fig. 9(a), we plot the temporal evolution of the total interface length in $2 \mathrm{~d}, \ell(t)$, (and averaged along the $z$-direction), as a function of time measured in $\mathrm{MC}$ steps. The temperature quench into the two-phase region is done for a fixed temperature, $T / J=2.0<T_{\mathrm{c}}(\lambda)$, in
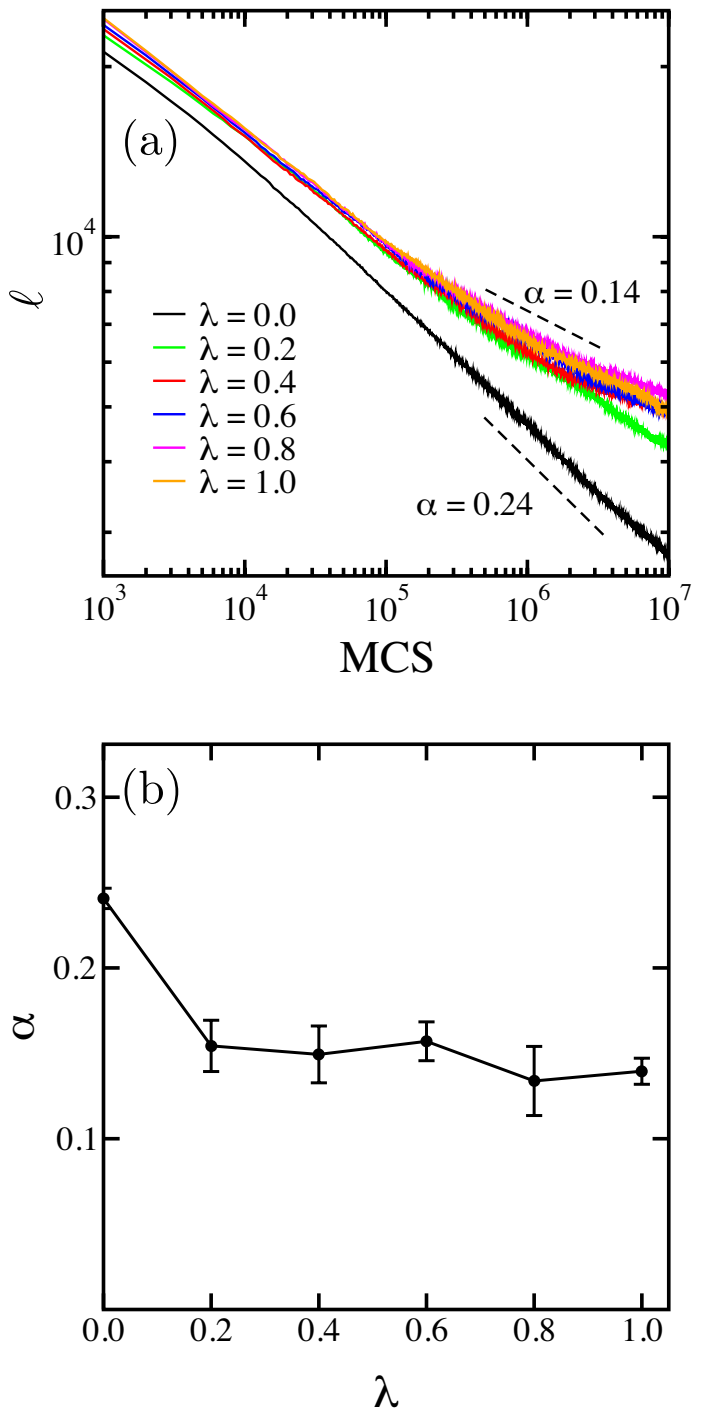

FIG. 10. The temporal evolution of the total interface length $\ell$ as a function of time (MCS) for different values of $\lambda=0$, $0.2,0.4,0.6,0.8,1.0$, and for a temperature quench from the one-phase state into the two-phase one, with final temperature satisfying $T=0.6 T_{\mathrm{c}}(\lambda)$. The A/B mixture is symmetric, $\bar{S}_{i, \rho}=$ $0, L=256$ and $L_{z}=8$. The average over three independent MC runs is taken for each $\lambda$ value. The two dashed lines represent a power-law behavior with exponents $\alpha=0.14$ and $\alpha=0.24$. (b) The domain growth exponent $\alpha$ as a function of $\lambda$, as obtained from (a).

order to mimic the experiment that is conducted at fixed room temperature. Several values of $\lambda$ are studied, and the other parameters are $L=256$ and $L_{z}=8$, with averages taken over three independent MC runs. For each $\lambda$ value, the scaling behavior of Eq. (9) is analyzed, and we extract the growth exponent $\alpha$ from the late stage kinetics. We find that for $\lambda=0$ (2d case), the growth exponent has the smallest value of $\alpha \approx 0.07$, while for $\lambda>0$, it is a function of $\lambda$ and increases up to $\alpha \approx 0.28$, as shown in Fig. 9(b). 
Although this result may explain the fact that the phase separation has an accelerated dynamics in stacked membranes as compared to GUVs (isolated single membranes), we should keep in mind that $T_{\mathrm{c}}(\lambda)$ increases as function of the inter-layer coupling $\lambda>0$, as shown in Fig. 6. As long as the final quench temperature is fixed to $T / J=2.0$, the temperature quench depth defined by $\Delta T=T_{\mathrm{c}}(\lambda)-T$ becomes larger as the value of $\lambda$ is increased. This may explain why the growth exponent $\alpha$ becomes larger with increasing $\lambda$, for a fixed $T$-quench.

In order to have a better comparison between different $\lambda$ values, we evaluate in Fig. 10 the growth exponent in a different way. We now keep a constant quench ratio $T / T_{\mathrm{c}}(\lambda)=0.6$, where $T$ is the final quench temperature, and the critical temperature $T_{\mathrm{c}}(\lambda)$ depends on $\lambda$, as shown in Fig. 6. For these deeper temperature quenches (farther from $T_{c}(\lambda)$ ), the estimated growth exponent is $\alpha \approx 0.24$ for $\lambda=0$ (pure 2d case), and $0.13 \leq \alpha \leq 0.16$ for $0.2 \leq \lambda \leq 1.0$. Note that the $\alpha$-values are only weakly dependent on $\lambda>0$.

Finally, we elaborate on the decreasing $\lambda$-dependence of the growth exponent $\alpha$, and show that this behavior is consistent with the change in the dimensionality of the stack from $2 \mathrm{~d}$ to $3 \mathrm{~d}$. In general, the growth exponent associated with phase separation depends on the dimensionality [23]. In this context, we mention the scaling argument of Binder and Stauffer on phase-separation dynamics of particles that undergo cluster reaction and diffusion processes [24]. Under the assumption that most particles that leave a cluster reimpinge on the same cluster at later times, the diffusion coefficient $D$ of a cluster of size $R$ was shown to scale as $D \sim R^{-(1+d)}$, where $d$ is the embedded space dimension. If we further assume that the domain size $R$ is the only length scale in the system, the scaling relation for a simple diffusion process is given by $R^{2} \sim D t$. This argument yields the growth exponent to be $\alpha=1 /(3+d)$. Hence, the predicted values from this scaling conjecture are $\alpha=1 / 5$ for $d=2$ and $\alpha=1 / 6$ for $d=3$.

Our simulation results, namely, $\alpha \approx 0.24$ for $\lambda=0$ and $\alpha \approx 0.14$ for $\lambda \geq 0.2$ compare favorably with this prediction. The growth exponent decreases for finite $\lambda$ because the system crosses-over from $2 \mathrm{~d}$ to $3 \mathrm{~d}$. This is due to the fact that the growing phase-separated domains are inter-connected along the $z$-direction for $\lambda>0$. It should be noted, however, that the absolute value of $\alpha$ obtained from the simulation is not universal but strongly depends on the quench depth as shown in Fig. 9. This explains why the above exponents are not in complete agreement with the simple scaling argument of Binder and Stauffer.

\section{CONCLUDING REMARKS}

Motivated by recent works of Tayebi et al. [5, 7], who studied experimentally and theoretically the phase separation in stacks of multi-component lipid bilayers, we have investigated the stacked $2 \mathrm{~d}$ Ising model given in Eq. (1). We use a Monte Carlo simulation scheme with Kawasaki exchange dynamics that conserves the order parameter in each layer, in order to investigate both equilibrium and dynamical features. Performing finitesize scaling analysis only in the lateral direction, while keeping the stack thickness fixed (mimicking the experiment), we determine the phase-transition temperature, $T_{\mathrm{c}}(\lambda)$, by changing the inter-layer interaction parameter $\lambda=J^{\prime} / J$. As shown in Fig. 6, the phase-transition temperature interpolates between that of the $2 \mathrm{~d}$ and $3 \mathrm{~d}$ Ising model.

One of our main conclusions is that domains in each one of the layers are always interconnected along the $z$ direction, forming a continuous columnar structure for any finite inter-layer interaction $J^{\prime}>0$, as shown in Fig. 5. This domain structure is in accord with the experimental findings for stacks of few dozen to few hundred layers [5]. However, the "multi-phase" region in which there are unaligned inplane domains with different composition, as was predicted in Ref. [7, is not found in our study at thermal equilibrium. Of course that such a "multi-phase" state can be transiently observed before the system reaches its fully equilibrated state, as can be observed in Figs. 2 and 5 .

We have also investigated the temporal evolution of domain formation in the stacked $2 \mathrm{~d}$ Ising model. When the inter-layer interaction $\lambda$ increases, the phase separation appears to have an accelerated dynamics as can be seen by the larger values of the growth exponent, $\alpha$, shown in Fig. 9(b). However, these larger $\alpha$ values are mainly due to an increase in the phase-transition temperature, $T_{\mathrm{c}}(\lambda)$, as function of $\lambda$; thus, a larger effective temperature quench, $\Delta T=T_{\mathrm{c}}(\lambda)-T$, for fixed $T$. When the final temperature quench $T$ is fixed relative to the phase-transition temperature as shown in Fig. 10 for $T=0.6 T_{\mathrm{c}}(\lambda)$, the growth exponent even decreases as the $\lambda$ value is increased. Our numerical findings for the growth exponent $\alpha$ are different than the value of $\alpha \approx 0.455$, as found in the experiment [5]. One possible explanation for this discrepancy can be the lack of hydrodynamic interactions in our MC simulations [6].

In this work, we have mainly discussed the case of $\bar{S}_{i, \rho}=0$, corresponding to the critical composition of the A/B lipid mixture. Currently, we are investigating the dynamics of phase separation for off-critical compositions, $\bar{S}_{i, \rho} \neq 0$ [see Fig. 5(b)]. For such compositions, the phase-transition temperature is smaller than the critical temperature. In the present simulations, the average A/B lipid composition (order parameter of the Ising model) in each bilayer is restricted to stay the same. In the future, we plan to study membrane stacks where each layer has a different but fixed composition [28]. Furthermore, since it is known from simulations that the presence of a supporting solid substrate affects the dynamics of membrane domain growth [29, it will be of interest to incorporate this substrate effect in future studies. 


\section{ACKNOWLEDGMENTS}

We thank H. T. Diep, J.-B. Fournier, T. Kato, R. Okamoto, H. Orland, P. Sens, S. Shimobayashi, M. Turner for useful discussions. T.H. acknowledges support of Tokyo Metropolitan University for an international student exchange program and the hospitality of Tel Aviv University. S.K. acknowledges support from the Grant-in-Aid for Scientific Research on Innovative Areas
"Fluctuation and Structure" (Grant No. 25103010) from the Ministry of Education, Culture, Sports, Science, and Technology of Japan, the Grant-in-Aid for Scientific Research (C) (Grant No. 24540439) from the Japan Society for the Promotion of Science (JSPS), and the JSPS Core-to-Core Program "International Research Network for Non-equilibrium Dynamics of Soft Matter". D.A. acknowledges support from the Israel Science Foundation under Grant No. 438/12 and the United States-Israel Binational Science Foundation under Grant No. 2012/060.
[1] B. Alberts, A. Johnson, P. Walter, J. Lewis, and M. Raff, Molecular Biology of the Cell (Garland Science, New York, 2008).

[2] G Schmitz and G Müller, J. Lipid Res. 32, 1539 (1991).

[3] S. L. Veatch and S. L. Keller, Biochim. Biophys. Acta 1746, 172 (2005).

[4] S. Komura and D. Andelman, Adv. Coll. Int. Sci. 208, 34 (2014).

[5] L. Tayebi, Y. Ma, D. Vashaee, G. Chen, S. K. Sinha, and A. N. Parikh, Nature Mater. 11, 1074 (2012).

[6] C. A. Stanich, A. R. Honerkamp-Smith, G. G. Putzel, C. S. Warth, A. K. Lamprecht, P. Mandal, E. Mann, T.-A. D. Hua, and S. L. Keller, Biophys. J. 105, 172 (2013).

[7] L. Tayebi, A. N. Parikh, and D. Vashaee, Int. J. Mol. Sci. 14, 3824 (2013).

[8] J. N. Israelachivili, Intermolecular and Surface Forces (Academic Press, New York, 2011).

[9] S. T. Milner and D. Roux, J. Phys. I France 2, 1741 (1992).

[10] S. Komura and D. Andelman, Europhys. Lett. 64, 844 (2003).

[11] K. W. Lee, J. Kor. Phys. Soc. 40, L398 (2002).

[12] M. J. de Oliveira, R. B, Griffiths, Surf. Sci. 71, 687 (1978).

[13] R. Pandit, M. Schick, and M. Wortis, Phys. Rev. B 26, 5112 (1982).

[14] K. Kawasaki, Phase Transitions and Critical Phenomena vol.2, edited by C. Domb and M. S. Green (Academic Press, NewYork, 1972).
[15] D. P. Landau and K. Binder, A Guide to Monte Carlo Simulations in Statistical Physics (Cambridge Univ. Press, New York, 2009).

[16] X. T. Pham Phu, V. Thanh Ngo, and H. T. Diep, Surface Science 603, 109 (2009).

[17] T. W. Capehart and M. E. Fisher, Phys. Rev. B 13, 5021 (1976).

[18] L. Onsager, Phys. Rev. 65, 117 (1944).

[19] K. Binder and E. Luijten, Phys. Rep. 344, 179 (2001).

[20] L. L. Liu and H. E. Stanley, Phys. Rev. Lett. 29, 927 (1972).

[21] M. Laradji and P. B. S. Kumar, Phys. Rev. Lett. 93, 198105 (2004); J. Chem. Phys. 123, 224902 (2005).

[22] S. Ramachandran, S. Komura, and G. Gompper, EPL 89, 56001 (2010).

[23] A. J. Bray, Adv. Phys. 51, 481 (2002).

[24] K. Binder and D. Stauffer, Phys. Rev. Lett. 33, 1006 (1974).

[25] A. B. Bortz, M. H. Kalos, J. L. Lebowitz, and M. A. Zendejas, Phys. Rev. B 10, 535 (1974).

[26] J. Marro, A. B. Bortz, M. H. Kalos, and J. L. Lebowitz, Phys. Rev. B 12, 2000 (1975).

[27] M. Rao, M. H. Kalos, J. L. Lebowitz, and J. Marro, Phys. Rev. B 13, 4328 (1976).

[28] K. Sornbundit, C. Modchang, N. Nuttavut, W. Ngamsaad, D. Triampo, and W. Triampo, J. Kor. Phys. Soc. 63, 71 (2013).

[29] W. Ngamsaad, S. May, A. J. Wagner, and W. Triampo, Soft Matter 7, 2848 (2011). 